\title{
Phase transformation in the alumina-titania system during flash sintering experiments
}

\author{
S. K. Jha, J. M. Lebrun and R. Raj \\ Department of Mechanical Engineering \\ University of Colorado at Boulder \\ Boulder, CO 80309-0427
}

September 2015

\begin{abstract}
We show that phase transformation in the alumina-titania system, which produces aluminum-titanate, follows an unusual trajectory during flash sintering. The experiments begin with mixed powders of alumina - titania and end in dense microstructures that are transformed into aluminum titanate. The sintering and the phase transformation are separated in time, with the sintering occurs during Stage II, and phase transformation during Stage III of the flash sintering experiment - Stage III is the steady-state condition of flash activated state that is established under current control, while Stage II is the period of transition from voltage to current control. The extent of phase transformation increases with the current density and the hold time in Stage III.
\end{abstract}

Corresponding author: Shikhar K. Jha

Email: shsh6593@colorado.edu 


\section{Introduction}

Electric field assisted sintering is a general description of at least three different processes, microwave sintering, spark plasma sintering (SPS) and, more recently, flash sintering. In SPS, very high power, in the range of several $\mathrm{kW}$, with high currents and low voltages, is used to rapidly heat a graphite die that contains the specimen. Simultaneously, a substantial pressure it applied with a punch. High rates of densification are obtained. Most importantly, ceramics such as yttria, silicon carbide and boron carbide, which are normally difficult to sinter, can be densified quickly in the SPS process. However, the complexity of the process makes it difficult to disentangle the role of the electric field, the heating rate and the applied pressure in the sintering process.

In flash sintering the electric field is applied directly to the specimen with a pair of electrodes. A conventional furnace is used. In contrast to SPS, flash sintering is a low power process with the power dissipation in the range of $10-1000 \mathrm{~mW} \mathrm{~mm}^{-3}$. The currents are in the $10-100 \mathrm{~mA} \mathrm{~mm}^{-2}$ range while the electric fields can range from $50 \mathrm{~V} \mathrm{~cm}^{-1}$ to $1000 \mathrm{~V} \mathrm{~cm}^{-1}$ $[1,2]$. The onset of the flash in accompanied by a highly non-linear rise in conductivity, which requires the power dissipation in the specimen to be controlled by switching from voltage to current control. Under current control, the specimen can be held nearly indefinitely in a constant state of flash, which is called Stage III. However, sintering occurs during the transient when the power is being switched from voltage to current control, which has been called Stage II. Several single-phase ceramics have been sintered in this way, often at temperatures that are far below, and time-periods that are much shorter than in conventional sintering [1-12]. Two-phase composites, including titania-alumina [14] and alumina-zirconia[15,16] have also been flash sintered.

In the present work, we show that flash sintering experiments also produce unusually high rates of a reaction between titania and alumina, to form aluminum titanate, a spinel structure having the composition $\mathrm{Al}_{2} \mathrm{O}_{3} \cdot \mathrm{TiO}_{2}$. This compound has a low coefficient of thermal expansion and a high melting point, attributes that make it useful for high temperature applications such as furnace linings. Aluminum titanate has been prepared by many routes, including hot pressing 
[17], combustion synthesis [18], solid state reaction with fine powders [19] and single crystals [20], ball milling [21], reaction sintering [22], and chemical routes [23].

According to the phase diagram, aluminum titanate forms above $1280{ }^{\circ} \mathrm{C}[24]$ as

$$
\alpha-\mathrm{Al}_{2} \mathrm{O}_{3}+\mathrm{TiO}_{2} \Leftrightarrow \beta-\mathrm{Al}_{2} \mathrm{TiO}_{5}
$$

In literature, there is evidence of the reaction taking place below $1280{ }^{\circ} \mathrm{C}$ when dopants such as $\mathrm{MgO}, \mathrm{SiO}_{2}, \mathrm{ZrO}_{2}$, or $\mathrm{Fe}_{2} \mathrm{O}_{3}$ are added [25-28].

Sol-gel processing [29] can also lower the temperature for the synthesis of aluminumtitanate. The compound has been made at temperatures as low as $600{ }^{\circ} \mathrm{C}[30]$ and $730{ }^{\circ} \mathrm{C}$ [31]. In this process, the constituents are molecularly mixed and form (metastable) pseudo $\beta-\mathrm{Al}_{2} \mathrm{TiO}_{5}$ which, on being heated to $1150{ }^{\circ} \mathrm{C}$, separates into alumina and titania. Our experiments are different: we show that alumina and titania can react and merge, forming the titanate phase under electrical fields at temperature where they are normally expected to remain as individual phases. A similar effect has been seen in SPS processing with powders prepared by the sol-gel process [32,33], where aluminum titanate has been shown to form at temperatures below the nomial phase transformation temperature of $1280{ }^{\circ} \mathrm{C}$.

\section{Experiments}

\subsection{Materials and Methods}

Titania (rutile) nanopowders (NP-TiO2-R-20 MTI Corporation Material Ltd., Richmond, CA) with average particle size $20 \mathrm{~nm}$ were mixed in with $20 \mathrm{vol} \%$ (18 wt \%) of alumina (dried form de-agglomerate alumina suspension, Allied High Tech Products, Inc., CA) of particle size $300 \mathrm{~nm}$. The details of powder preparation has been described elsewhere [14]. Powders were uniaxially pressed into dog-bone shapes [2] under a pressure of $105 \mathrm{MPa}$. Average dimensions of the gage-section of the dog-bones were measured to be $19.8 \mathrm{~mm}$ x $3.5 \mathrm{~mm}$ x $1.1 \mathrm{~mm}$. Specimen were hung into the middle of the furnace with the help of platinum wires which also acted as electrodes for applying electric field.

A 3000 W DC power supply (Glassman 2000V-1.5A, Glassman High voltage, Inc. NJ, USA) was used to apply the electric field. A digital multimeter (Keithley 2000; Keithley 
Instruments, Cleveland, $\mathrm{OH}$ ) was connected in series to measure the current flowing through the specimen. Samples were first pre-sintered for $1 \mathrm{~h}$ at $550^{\circ} \mathrm{C}$ to burn off the binder. The pre-

sintered specimens were heated at $10^{\circ} \mathrm{C} \mathrm{min}^{-1}$ up to $830^{\circ} \mathrm{C}$ at and held at this temperature for 100 $\mathrm{s}$ before applying a DC voltage corresponding to $500 \mathrm{~V} \mathrm{~cm}^{-1}$.

A typical example of flash sintering experiment for $\mathrm{TiO}_{2}-\mathrm{Al}_{2} \mathrm{O}_{3}$ composite is shown in Fig. 1. The flash initiated after an incubation period of $18 \mathrm{~s}$. At this point, the power supply was switched to current control. Under current-control, the specimen could be maintained in a constant state of flash. The incubation period is called Stage I, the transient to current control, Stage II, and the steady state of flash, Stage III. The power density is the product of the field and the current density. The peak-height in the power spike is ideally the product of these two variables in the experiment. After the peak, the power density is determined by the voltage that develops across the specimen in the flash state under current control.

The principal control variables in flash sintering experiments are the furnace temperature, and the electric field, in Stages I and II, and the current setting and the hold time in Stage III. The present experiments were carried out at a furnace temperature of $830^{\circ} \mathrm{C}$, with a DC field of 500 $\mathrm{V} \mathrm{cm}^{-1}$. The current density and the hold time, in Stage III, were varied to study their influence on sintering and phase transformation. The ranges of these two variables are given in Fig. 2. Point ' $A$ ' refers to the flash experiment reported in Fig. 1. Regime B shows the range of current density; in these experiments, the hold time was kept constant at $60 \mathrm{~s}$. In Regime C, the current density was held at $30 \mathrm{mAmm}^{-2}$, while the hold time was varied from $10 \mathrm{~s}$ to $300 \mathrm{~s}$.

The sample temperature in Stage III rises above the furnace temperature due to Joule heating. It was measured from in-situ measurements of thermal expansion at the APS at Argonne National Laboratory, and also estimated from a black body radiation model. The procedure followed at the APS is described below.

\subsection{Quantitative measurement of the phase transformation}

The relative intensity ratio (RIR) method in X-ray diffraction [34] was employed to measure the conversion of alumina and titania into aluminum titanate. Silicon was used as the internal reference, since its prime peak (111) does not overlap with titania, alumina or the 
aluminum titanate phase. Silicon powder of a weight equal to that of specimen were mixed and ground together. The relative strengths of the diffraction peaks from silicon and aluminum oxide were used to quantify the formation of the aluminum titanate.

$\mathrm{X}$-ray diffraction measurements were performed with $\mathrm{CuK}_{\alpha}$ radiation $\left(\lambda=1.5406 \mathrm{~A}^{\circ}\right)$ in a D2 PHASER $2^{\text {nd }}$ GEN (Bruker AXS GmbH, Karlsruhe, Germany) system. The scans were done in $2 \theta$ range of $20^{\circ}-50^{\circ}$ with a step size of $0.01^{\circ}$ and acquisition time of $1 \mathrm{~s}$ per step. Bruker Diffract Suite Eva software was used to fit a Gaussian curve to the XRD scans and remove the background noise before comparing the peaks intensities.

The phase transformation is characterized by the emergence of the (101) diffraction peak for aluminum-titanate which occurs at $2 \theta=26.6^{\circ}$ (for $\lambda=1.5406 \mathrm{~A}^{\circ}$ ), and the concomitant decline in the (012) peak of alumina, at $2 \theta=25.6^{\circ}$, as it is consumed to form the spinel. The phase transformation was quantified by measuring the weight fraction of alumina in the specimen using the silicon standard. For this purpose powders of alumina and silicon were mixed with alumina content ranging from $5 \%$ to $20 \%$ by weight; the relative strengths of the alumina (102) peak and silicon (111) peak were measured to obtain a calibration curve, which is given in Fig. 3. The error bar was obtained from the dispersion in the data.

\subsection{In-situ X-ray diffraction experiments to measure specimen temperature and the rate of phase transformation in real time}

In-situ flash experiments were conducted at the APS at Argonne National Laboratory to measure (i) the specimen temperature, and (ii) phase transformation. The schematic of this experimental set up is shown in Fig. 4. Rectangular bars of average physical dimension $3.8 \mathrm{~mm} \mathrm{x}$ $1.3 \mathrm{~mm}$ x $0.7 \mathrm{~mm}$ were uniaxially pressed under $100 \mathrm{MPa}$. Electric field of $500 \mathrm{Vcm}^{-1}$ was applied to the specimen through the platinum wires wrapped around the two ends of the gagelength. Sorensen 300-2, Sorensen San Diego, CA was used as the DC power source and a digital multimeter (Keithley 2000, Keithley instruments, Cleveland, OH) was connected in series to measure the current flowing through the specimen. The experiments were carried out on beamline 33BMC X-Ray beamline at a radiation wavelength of $0.59^{\circ} \mathrm{A}$. The set up allowed the diffraction in transmission mode and the spectra was measured with a Pilatus 100K 2D image plate detector (DECTRIS Ltd, Villigen, Switzerland) kept on the other side of the specimen as 
shown in Fig. 4 (b). Diffraction data were obtained with a fixed detector position, which images the diffraction pattern with a $2 \theta$ (the Bragg angle) ranging from $12.2^{\circ}$ to $16.4^{\circ}$. This range includes the diffraction peaks from alumina (plane (104) PDF-421468), titania (plane (110) PDF 890555), aluminum titanate (plane (023) PDF-810030) and platinum (plane (111), PDF-040802) which was painted on the surface as reference temperature standard (explained later). Acquisition rate was one scan per sec to get reasonably good diffraction pattern. A quadrupole Lamp furnace [35] was used in these experiments; it allowed the X-ray to enter and diffract through the specimen without interacting with the furnace. In a typical run, the specimen temperature was raised to $900{ }^{\circ} \mathrm{C}$ and a field of $500 \mathrm{Vcm}^{-1}$ was applied as a step function. The current limit was set at $20 \mathrm{~mA} \mathrm{~mm}^{-2}$. It was later increased to $25 \mathrm{mAmm}^{-2}$ to study the effect of change in current on the kinetics of the reaction. The reduction in the intensity of the alumina peak was taken as the measure of the extent of phase transformation.

Platinum was used as the standard to measure the surface specimen temperature. A sliver of platinum paste was applied on one side of the specimen. The specimen was heated at different furnace temperatures (without electric field) and the corresponding shift in the (111) Pt peak was measured. Thermal expansion data for Pt was then used to correlate the peak shift to the specimen temperature, which led to the calibration curve given in Fig. 5. In live experiments, the Pt peak shift was measured and the specimen temperature estimated from this calibration curve.

\subsection{Estimate of temperature from the black body radiation model and comparison with direct measurement from the in-situ experiments at APS}

The specimen temperature can be calculated from electrical power dissipated in the sample by equating it to black body radiation (BBR),

$$
\frac{T^{*}}{T_{o}}=\left[1+\frac{1000 \cdot W_{v}}{e_{m} \sigma T_{o}^{4}}\left(\frac{V}{A}\right)\right]^{\frac{1}{4}}
$$

The specimen temperature, $T^{*}$ is expressed in terms of furnace temperature $T_{o}(\mathrm{~K})$ and $W_{v}$, power dissipation per unit volume $\left(\mathrm{mW} \mathrm{mm}^{-3}\right) . \sigma=5.67 \times 10^{-8} \mathrm{Wm}^{-2} \mathrm{~K}^{-4}$ is the StefanBoltzmann constant and $e_{m}$ is the emissivity ratio, typically 0.9 for ceramics. The volume to surface ratio is given by (V/A). It is calculated to be $0.25 \mathrm{~mm}$ for the specimens used at the 
synchrotron. For the dog-bone specimens in experiments carried out in our own laboratory in Boulder, Colorado, this ratio is equal to $0.45 \mathrm{~mm}$.

The temperature of specimen measured in Stage III with the Pt standard and computed from the BBR model, are compared in Table1. The experimental data were obtained at two current densities, $20 \mathrm{mAmm}^{-2}$, and $25 \mathrm{mAmm}^{-2}$. The BBR estimates agree with the direct measurements with the platinum standard to within $\pm 25^{\circ} \mathrm{C}$.

Table 1 Black body radiation temperature calculations and comparison with in-situ measurements.

\begin{tabular}{|c|c|c|c|c|}
\hline \multicolumn{2}{|c|}{ Flash parameters } & \multirow{2}{*}{$\begin{array}{l}\text { Furnace } \\
\text { temp. } \\
\left({ }^{\circ} \mathrm{C}\right)\end{array}$} & \multicolumn{2}{|c|}{$\begin{array}{l}\text { Estimated specimen } \\
\text { temperature }\end{array}$} \\
\hline Electric field & Current density & & $\operatorname{BBR}\left({ }^{\circ} \mathbf{C}\right)$ & In situ $\left({ }^{\circ} \mathbf{C}\right)$ \\
\hline $500 \mathrm{~V} / \mathrm{cm}$ & $20 \mathrm{mAmm}^{-2}$ & $900{ }^{\circ} \mathrm{C}$ & $1150 \pm 10$ & $1180 \pm 30$ \\
\hline $500 \mathrm{~V} / \mathrm{cm}$ & $25 \mathrm{mAmm}^{-2}$ & $900^{\circ} \mathrm{C}$ & $1310 \pm 15$ & $1340 \pm 30$ \\
\hline
\end{tabular}

Strictly speaking, the platinum measures the surface temperature of the specimen. It is possible that the temperature within the specimen is higher. This difference, between the temperature within the mid-section of the specimen and its surface can be estimated from the equation for heat transport, given by

$$
\frac{d Q}{d t}=-k \cdot A \cdot \frac{d T}{d x}
$$

Here $\frac{d Q}{d t}$ is the heat flowing from the interior of the specimen to the surface, $\frac{d T}{d x}$ is the temperature gradient, $k$ is the thermal conductivity of the specimen $\left(0.0038 \mathrm{~W} . \mathrm{K}^{-1} \mathrm{~mm}^{-1}\right.$, applying rule of mixture for composite [36]). $A$ is the cross-section area of the specimen which was equal to $15.2 \mathrm{~mm}^{2}$. The distance from the mid-section to the surface of the specimen, that is one-half the specimen thickness was $0.35 \mathrm{~mm}$. The heat flow through the specimen must necessarily be equal to the electrical power dissipation within the specimen. The upper bound in 
the temperature difference between the specimen center and its surface is calculated by assuming that all the power is dissipated in the middle of the specimen. Even with this extreme assumption, we calculate a difference in the temperature within the interior and the surface to be only $7{ }^{\circ} \mathrm{C}$ (the power dissipation was $1.14 \mathrm{~W}$ ). More realistically, the power is dissipated throughout the entire cross section of the specimen and, therefore, the difference between the interior and the surface will be much less than $7^{\circ} \mathrm{C}$.

The absence of a measureable difference in temperature within the specimen was also verified by Terauds et al. [37] where a one-to-one correlation between the expansion in the platinum film and the expansion in the bulk of the zirconia specimen, was established.

\subsection{Microstructure}

The microstructure of the specimen, flash sintered at a current limit of $25 \mathrm{~mA} \mathrm{~mm}^{-2}$ with a hold time of $600 \mathrm{sec}$ (in-situ experiment), was examined. The specimen was cut in the middle of the gage section, polished down to $1-\mu \mathrm{m}$ diamond paste and then thermally etched at $900{ }^{\circ} \mathrm{C}$ for 1 hour. Low vacuum SEM (JEOL JSM-6480LV, Tokyo, Japan) was used to obtain the microstructure. The phases were identified by their composition using the EDS attachment.

\section{Results}

\subsection{Flash sintering of $80 / 20 \mathrm{vol} \% \mathrm{TiO}_{2}-\mathrm{Al}_{2} \mathrm{O}_{3}$ composite}

The flash sintering behavior of this composite at a current density of $15 \mathrm{~mA} \mathrm{~mm}{ }^{-2}$ with hold time of $20 \mathrm{~s}$ was shown in Fig. 1. It is marked as point A in Fig. 2. The linear shrinkage is synchronized with the curve for the power density. Note that densification is enhanced by the electric field before the onset of the flash, that is, in Stage I. Approximately one half of the densification is achieved in Stage I, and the remainder within $5 \mathrm{~s}$ after the flash. The final density of this specimen, measured by the Archimedes method was $92 \%$. Note that the power curve, which is the product of electric field and current rises quickly at the end of Stage I (voltage controlled), reaching its peak when the power supply switches to current control, and then reaching a plateau in Stage III. 


\subsection{In-Situ Experiments at the APS: separation of sintering and phase transformation}

We find that sintering and phase transformation occur during different periods of the flash experiment. Densification occurs predominantly in Stage II, whereas the phase transformation begins in Stage III. This experiment was done at a furnace temperature of $900^{\circ} \mathrm{C}$. The flash was initiated with a DC field of $500 \mathrm{~V} \mathrm{~cm}^{-1}$. Initially the current limit was set at $20 \mathrm{~mA}$ $\mathrm{mm}^{-2}$ for a period of $250 \mathrm{~s}$, and then increased, in the same experiment, to $25 \mathrm{~mA} \mathrm{~mm} \mathrm{~m}^{-2}$ in order to enhance the kinetics of the reaction.

The results in Fig. 6 give diffraction data from live in-situ experiments; they show the gradual decline of the alumina peak and the complementary rise in the peak from the emerging phase of aluminum titanate. Note that the spinel peak begins to appear at approximately $150 \mathrm{~s}$. At $600 \mathrm{~s}$ the alumina peak has nearly dissolved while the aluminum titanate peak has grown fully.

Current density has shown to have huge effect on the kinetics. (A movie showing the relative change in the peak strengths of alumina and aluminum titanate in a local X-ray diffraction scan at a current density of $25 \mathrm{~mA} \mathrm{~mm}^{-2}$ is included as Supplementary Material).

Microstructure of the specimen at the end of the reaction is shown in Fig. 7 along with EDS pattern from the titania rich phase and the aluminum titanate phase. No pure alumina phase was seen.

One may think that the multiphase microstructure may cause distortion in the local current flow. That can happen when the discontinuity is extreme, as for example when large inclusions of alumina $(10 \mu \mathrm{m})$ are co-sintered in a titania matrix using flash sintering [14]. In that instance the agglomerates retained a large porosity within, which prevented the current from flowing through them which caused inhomogeneous sintering. However, in the present composites the microstructural length scale is much smaller. We did not expect, and nor did we see any discontinuities in the evolution of the microstructure and sintering.

In summary, we note that while both sintering and phase transformation are accelerated in flash experiments, they occur at different time scales. Densification occurs predominantly during Stage II, but phase transformation occurs later in Stage III. The phase transformation with 
and without the electric field is explored in detail in the next section. It should be notes that sintering requires stoichiometric diffusion while phase transformations can occur with the transport of just one charged species, the one that is faster moving.

\subsection{The influence of power variables on the kinetics of phase transformation}

The state of the specimen in Stage III is determined by the current density set at the power supply. The temperature of the specimen is self-determined through a steady state established by power dissipation and black body radiation. If the temperature rises above this steady state value, then the resistance falls and the $I^{2} R$ heating drops, causing the specimen to cool. If the temperature falls below the equilibrium temperature then the resistance rises causing greater power dissipation and the specimen temperature rises back to its equilibrium value. This steady state temperature is calculated from Eq. (2).

Our approach has been to measure the phase transformation as a function of the hold time at different specimen temperatures, which are controlled by the current density. These data are compared with transformation kinetics under conventional conditions by conducting experiments without the electric field at furnace temperature that is set equal to the specimen temperature of the field-assisted experiments.

The data for phase transformation with and without the electric field, according to the above protocol are shown in Fig. 8. The extent of the phase transformation is characterized by the depletion of the alumina phase, which is measured quantitatively by X-ray diffraction with the silicon standard.

The plot on the left in Fig. 8(a) shows the depletion in alumina in experiments with three specimens, one held at $60 \mathrm{~s}$ with a current density of $20 \mathrm{~mA} \mathrm{~mm}{ }^{-2}$, the second for $60 \mathrm{~s}$ with 30 $\mathrm{mA} \mathrm{mm} \mathrm{m}^{-2}$, and the third for $60 \mathrm{~s}$ at a current density of $40 \mathrm{~mA} \mathrm{~mm}{ }^{-2}$. The specimen temperatures calculated from the BBR model, using Eq. (2), are plotted along the abscissa. The downward sloping nature of the trend suggests that the rate of the reaction rises non-linearly with the specimen temperature, akin to Arrhenius behavior.

The right hand plot in Fig. 8(b) compares the reaction kinetics with and without the

electric field. At a current density of $30 \mathrm{~mA} \mathrm{~mm}^{-2}$ the reaction nears completion in $300 \mathrm{~s}$ (from 
the onset of the flash). At this current density, the specimen temperature is estimated to be 1250 ${ }^{\circ} \mathrm{C}$. However, without the electric field alumina-titania composite shows negligible reaction at a furnace temperature of $1250{ }^{\circ} \mathrm{C}$ even after 5 hours. This result is clear evidence of field induced phase transformation. Experiments carried out at furnace temperature of $1450{ }^{\circ} \mathrm{C}$, without the electric field, show some reaction but again far less than under the electric field at a far lower specimen temperature.

\subsection{Phase transformation in real time measured at the APS synchrotron}

In-situ experiments at the APS provided measurement of the phase transformation in real time. We plot the area under the alumina peak, normalized with respect to its initial value, as a function of time (refer to Supplementary material: video of changing peak intensities of alumina and aluminum titanate with flash). The current density and therefore, the specimen temperature was kept constant at $1250{ }^{\circ} \mathrm{C}$. A logarithmic plot of the alumina intensity versus time is given in Fig 9. The reaction begins after a brief incubation period of $50 \mathrm{~s}$, which we ascribe to the nucleation of the new phase. Thereafter the data show a $t^{n}$ dependence of the reaction rate where $n \quad 0.5$. We have also measured the rate of the reaction without the field with the specimen being held at $1500{ }^{\circ} \mathrm{C}$; these result show as similar value for $n$.

In order for the $\mathrm{Al}_{2} \mathrm{TiO}_{5}$ phase to grow, either $\mathrm{Ti}^{4+}$ or $\mathrm{Al}^{3+}$ must diffuse through the new phase to react with the other constituent. The faster moving species will control the reaction kinetics. The formation of $\mathrm{Al}_{2} \mathrm{TiO}_{5}$ in bicrystals and with fine powers of alumina and titania, the reaction was determined by Freudenberg and Mocellin [20] to be diffusion controlled. In a parallel paper the solid-state reaction in fine alumina and titania powders $[19,20]$ was also determined to be diffusion controlled. These reactions followed parabolic kinetics, where $n=0.5$ [20]. It was determined that $\mathrm{Al}^{3+}$ ions diffused through the aluminum titanate phase to react with titania.

\section{Discussion}

Flash experiments consist of three stages in experiments carried out at a constant furnace temperature. Stage I is the incubation time between the application of the field and the onset of 
the non-linear rise in conductivity. Stage II is the transient during which the power supply is switched from voltage to current control. Stage II, which lasts less than five seconds, is the period during which samples sinter. Stage III is the steady state of flash established under current control.

Experiments in Stage III, because they are long-lived, are particularly amenable for insitu measurements of X-ray diffraction at the synchrotron. In earlier work with 3 mol\% yttria stabilized zirconia (3YSZ), we have discovered the emergence of a new phase which rises and extinguishes when the field is turned on and off [38]. In experiments with titania we have seen the strengthening of some peaks and simultaneously weakening of other peaks under the electric field [39]. These are intriguing results that show that the effect of the flash extends beyond sintering.

The present experiments illustrate that the flash effect also greatly accelerates the reaction between alumina and titania for the formation of aluminum-titanate. We further note that this reaction occurs at a temperature that is below the normal phase diagram temperature for the formation of aluminum-titanate. A similar effect has been observed when powders prepared by sol-gel are sintered by spark-plasma-sintering [32, 33].

A rapid rise in conductivity is a signature of the flash event. While the event is instigated by an applied field, once it initiates the power dissipation in the specimen must be limited by switching from voltage to current control. Power dissipation produces Joule heating. Thus, there is always a question whether the events in flash experiments are simply a result of Joule heating or whether they are instigated by the electrical field.

Thus, we have been careful to determine the specimen temperature from thermal expansion, which is measured during in-situ experiments at the APS at the Argonne National Laboratory. A platinum standard is used to calibrate the thermal expansion of the ceramic phase. We then conduct experiments without the electric field with the specimens being heated to the same temperature as determined from the in-situ measurements during Joule heating. In this way, we are able to rule out that Joule heating is the sole cause of these accelerated kinetics and unusual phase transformations. 
It is interesting that flash sintering and the phase transformation in the alumina-titania system occur at different time scales. Whereas sintering occurs during Stage II in a very short time, typically less than five seconds, the chemical reaction occurs during the hold time in Stage III. Whether this delay reflects the difference in the diffusion mechanism - sintering is controlled by self-diffusion while phase change occurs by the diffusion of a charged species - or a difference in the diffusion pathway is difficult to say.

\section{Conclusions}

It is conclusively shown that a reaction between $\mathrm{Al}_{2} \mathrm{O}_{3}$ and $\mathrm{TiO}_{2}$ to form $\mathrm{Al}_{2} \mathrm{TiO}_{5}$ is significantly accelerated by electrical fields.

The above conclusion is reached in three steps: (i) the specimen temperature in Stage III is measured by in-situ measurements of thermal expansion at the Advanced Photon Source at the Argonne National Laboratory, (ii) the kinetics of phase transformation is measured in Stage III, and (iii) the results are compared to phase transformation without the electric field at furnace temperature set equal to the specimen temperatures measured at the synchrotron. From these measurements we find that field assisted phase transformation is nearly complete in $300 \mathrm{~s}$, when

the specimen is at $1250{ }^{\circ} \mathrm{C}$. Specimens heated to this temperature without the electric field barely show any phase transformation even after $5 \mathrm{~h}$.

The results highlight that flash experiments not only achieve ultrafast sintering, they also accelerate kinetics of chemical reactions, above and beyond Joule heating. These results augment other work where we have shown that new phases and texture can develop and extinguish when the electric field is turned on and off $[38,39]$.

\section{Acknowledgements}

It is a pleasure to acknowledge Prof. W. Kriven and her group for their help, especially in the use of the high temperature lamp furnace at APS. We are pleased to acknowledge assistance and discussion with Evguenia Karapetrova and Christian M. Schlepuetz, physicists at Argonne National Lab, Beamline 33BMC where the in-situ synchrotron experiments were performed. Use 
of the Advanced Photon Source was supported by the U. S. Department of Energy, Office of Science, Office of Basic Energy Sciences, under Contract No. DE-AC02-06CH11357. Lebrun was supported in part by a seed grant from the Army Research Office, W911 NF-14-1-0569, and more substantially by a grant from the Office of Naval Research under the direction of Dr. Lawrence Kabacoff and Dr. Antti Makinen, under the grant number N00014-12-1-0710. Raj and Jha, and the development of facilities for these flash experiments at the University of Colorado were supported by the Basic Energy Science Division of the Department of Energy under grant number DE-FG02-t07ER46403.

\section{References}

[1] Cologna M, Rashkova B, Raj R. Flash sintering of nanograin zirconia in $<5 \mathrm{~s}$ at $850 \mathrm{C}$. J Am Ceram Soc 2010;93:3556-9.

[2] Cologna M, Francis JSC, Raj R. Field assisted and flash sintering of alumina and its relationship to conductivity and MgO-doping. J Eur Ceram Soc 2011;31:2827-37.

[3] Karakuscu A, Cologna M, Yarotski D, Won J, Francis JSC, Raj R, et al. Defect Structure of Flash-Sintered Strontium Titanate. J Am Ceram Soc 2012;95:2531-6.

[4] Prette ALG, Cologna M, Sglavo V, Raj R. Flash-sintering of Co2MnO4 spinel for solid oxide fuel cell applications. J Power Sources 2011;196:2061-5.

[5] Jha SK, Raj R. The Effect of Electric Field on Sintering and Electrical Conductivity of Titania. J Am Ceram Soc 2014;97:527-34.

[6] Yoshida H, Sakka Y, Yamamoto T, Lebrun J-M, Raj R. Densification behaviour and microstructural development in undoped yttria prepared by flash-sintering. J Eur Ceram Soc 2014;34:991-1000.

[7] Muccillo R, Muccillo ENS. Electric field-assisted flash sintering of tin dioxide. J Eur Ceram Soc 2014;34:915-23.

[8] Liu Y, Hao X, Wang Z, Wang J, Qiao J, Yan Y, et al. A newly-developed effective direct current assisted sintering technique for electrolyte film densification of anode-supported solid oxide fuel cells. J Power Sources 2012;215:296-300.

[9] Gaur A, Sglavo VM. Flash-sintering of $\mathrm{MnCo} 2 \mathrm{O} 4$ and its relation to phase stability. J Eur Ceram Soc 2014; 34:2391-2400. 
[10] Zhang Y, Jung J-I, Luo J. Thermal runaway, flash sintering and asymmetrical microstructural development of $\mathrm{ZnO}$ and $\mathrm{ZnO}-\mathrm{Bi} 2 \mathrm{O} 3$ under direct currents. Acta Mater 2015;94:87-100.

[11] Bonilla S, Wilshaw PR, Todd RI. Preliminary investigation of flash sintering of SiC. J Eur Ceram Soc 2013;33:2811-6.

[12] Grasso S, Saunders T, Porwal H, Cedillos-Barraza O, Jayaseelan DD, Lee WE, et al. Flash Spark Plasma Sintering (FSPS) of Pure ZrB2. J Am Ceram Soc 2014;97:2405-8.

[13] Shomrat N, Baltianski S, Randall C a., Tsur Y. Flash sintering of potassium-niobate. J Eur Ceram Soc 2015;35:2209-13.

[14] Jha SK, Raj R. Electric Fields Obviate Constrained Sintering. J Am Ceram Soc 2014;97:3103-9.

[15] Bichaud E, Chaix JM, Carry C, Kleitz M, Steil MC. Flash sintering incubation in Al2O3/TZP composites. J Eur Ceram Soc 2015; 35:2587-2592.

[16] Naik KS, Sglavo VM, Raj R. Field assisted sintering of ceramic constituted by alumina and yttria stabilized zirconia. J Eur Ceram Soc 2014;34:2435-42..

[17] Stamenkovic I. Aluminium Titanate-Titania Ceramics Synthesized by Sintering and Hot Presstng. Ceram Int 1989;15:155-60.

[18] Segadges AM. Combustion Synthesis of Aluminium Titanate. J Eur Ceram Soc 1998;18:771-81.

[19] Freudenberg B, Mocellin A. Aluminum Titanate Formation by Solid-state Reaction of Fine A1203 and Ti02 Powders. J Am Ceram Soc 1987;70:33-8.

[20] Freudenberg B, Mocellin A. Aluminium titanate formation by solid state reaction of AI203 and TiO2 single crystals. J Mater Sci 1990;25:3701-8.

[21] Uribe R, Baudin C, Mazerolles L, Michel D. Sub-micron sized Al 2 TiO 5 powders prepared by high-energy ball milling. J Mater Sci 2001;36:5105-13.

[22] Buscaglia V, Nanni P, Battilana G, Aliprandi G, Carry C. Reaction sintering of aluminium titanate: II—Effect of different alumina powders. J Eur Ceram Soc 1994;13:419-26.

[23] Okamura H, Barringer EA, Bowen HK. Preparation and Sintering of Monosized A12O3TiO2 Composite Powder. J Am, Ceram Soc, 1986;69:22-4.

[24] Kato E, Daimon K, Takahashi J. Decomposition temperature of beta Al2TiO5. J Am Ceram Soc 1980:355-6. 
[25] Buscaglia V, Battilana G, Leoni M, Nanni P. Decomposition of A12TiO5-MgTi2O5 solid solutions: a thermodynamic approach. J Mater Sci 1996;31:5009-16.

[26] Buscaglia V, Alvazzi Delfrate M, Leoni M, Bottino C, Nanni P. The effect of MgA12O4 on the formation kinetics of $\mathrm{A} 2 \mathrm{TiO} 5$ from $\mathrm{Al} 2 \mathrm{O} 3$ and $\mathrm{TiO} 2$ fine powders. J Mater Sci 1996;31:1715-24.

[27] Korim $\mathrm{T}$. Effect of $\mathrm{Mg} 2+-$ and $\mathrm{Fe} 3+-$ ions on formation mechanism of aluminium titanate. Ceram Int 2009;35:1671-5.

[28] Ishitsuka M, Sato T, Endo T, Shimada M. Synthesis and Thermal Stability of Aluminum Titanate Solid Solutions. J Am Chem Soc 1987;70:69-71.

[29] Vioux a. Nonhydrolytic Sol - Gel Routes to Oxides Nonhydrolytic Sol - Gel Routes to Oxides 1997;9:2292-9.

[30] Andrianainarivelo M, Corriu R, Leclercq D, Mutin P, Vioux a. Nonhydrolytic sol-gel process: aluminum titanate gels. Chem Mater 1997;9:1098-102.

[31] Bonhomme-Coury L, Lequeux N, Mussotte S, Boch P. Preparation of Al2TiO5 - ZrO2 Mixed Powders via Sol-Gel Process. J Sol-Gel Sci Tech 1994;375:371-5.

[32] Stanciu L, Groza JR, Kodach VY, Crisan M, Zaharescu M. Electric-field effects on sintering and reaction to form aluminum titanate from binary alumina-titania sol-gel powders. J Am Ceram Soc 2001;84:983-5.

[33] Stanciu L, Groza JR, Stoica L, Plapcianu C. Influence of powder precursors on reaction sintering of Al 2TiO5. Scr Mater 2004;50:1259-62.

[34] Terauds K. Processing, Structure and High Temperature Oxidation Properties of Polymer-Derived and Hafnium Oxide Based Ceramic Systems. Ph.D. Thesis, University of Colorado, Boulder, 2014.

[35] Sarin P, Yoon W, Jurkschat K, Zschack P, Kriven WM. Quadruple Lamp Furncae for High Temperature (up to $2050 \mathrm{~K}$ ) Synchrotron Powder X-ray Diffraction studies in Air in Reflection Geometry. Rev Sci Instrum, 2006;77:093906.

[36] Kingery WD, Francl J, Coble RL, Vasilos T. Thermal conductivity: X, data for several Pure Oxide Material Correlated to Zero Porosity. J Am Ceram Soc; 37: 107-10.

[37] Terauds K, Lebrun J-M, Lee H-H, Jeon T-Y, Lee S-H, Je JH, et al. Electroluminescence and the measurement of temperature during Stage III of flash sintering experiments. J Eur Ceram Soc 2015;35:3195-9. 
[38] Lebrun J-M, Morrissey TG, Francis JSC, Seymour KC, Kriven WM, Raj R. Emergence and Extinction of a New Phase During On-Off Experiments Related to Flash Sintering of 3YSZ. J Am Ceram Soc 2015:98: 1493-97.

[39] Jha SK, Lebrun JM, Seymour KC, Kriven WM, Raj R. Electric Field Induced Texture in Titania during Experiments Related to Flash Sintering. J Eur Ceram Soc 2015. Accepted. doi 10.1016/j-jeurceramsoc.2015.09.002. 


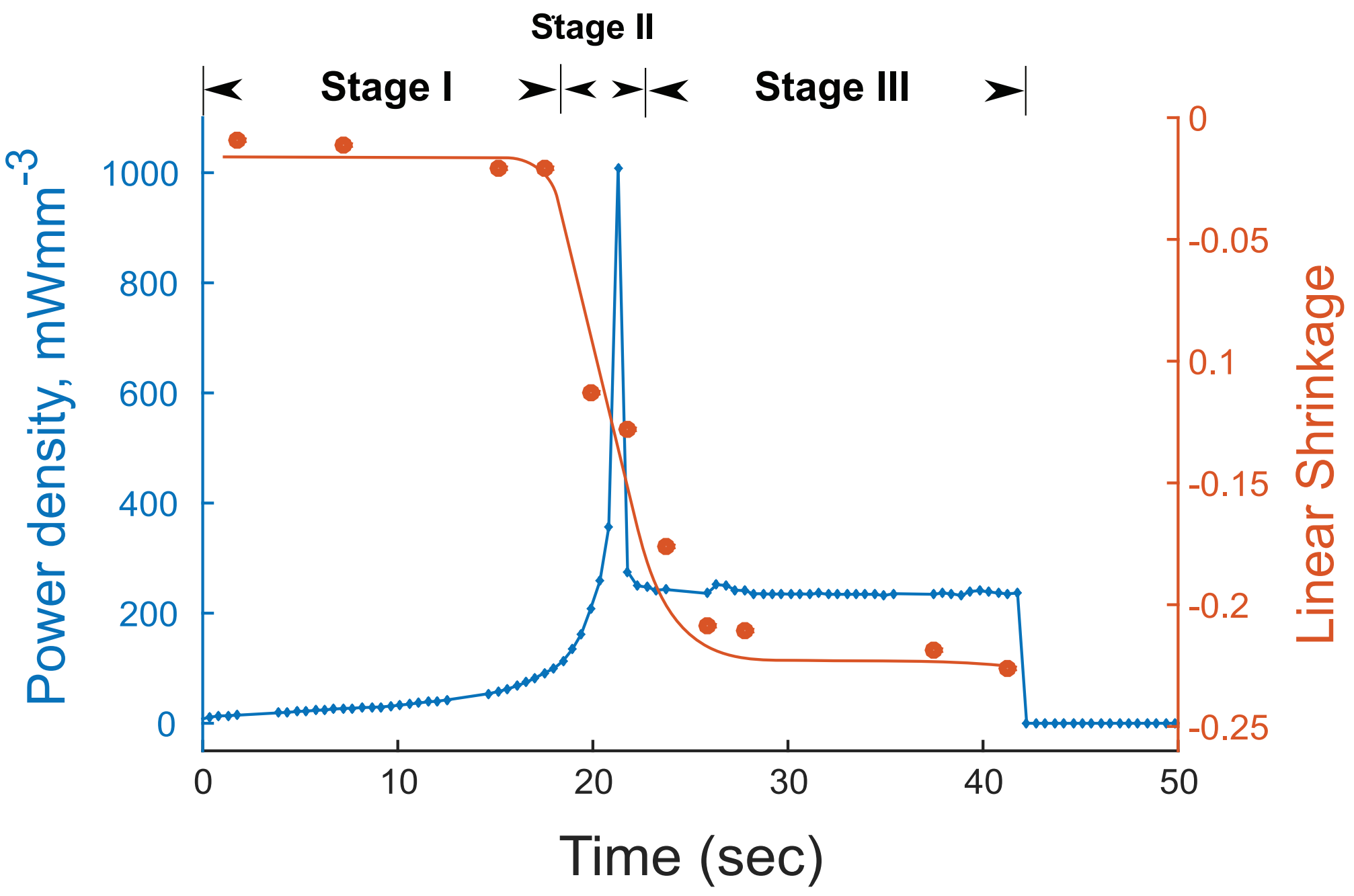

Figure 1. A typical flash sintering experiment for composite $\mathrm{TiO} 2-\mathrm{Al} 2 \mathrm{O} 3$ at a current density of $15 \mathrm{~mA} \mathrm{~mm}^{-2}$. Stage I is the incubation stage, under voltage control, before the onset of the flash, the transient is Stage II during which time the sample sinters, and the following steady state of flash under current control is Stage III. 


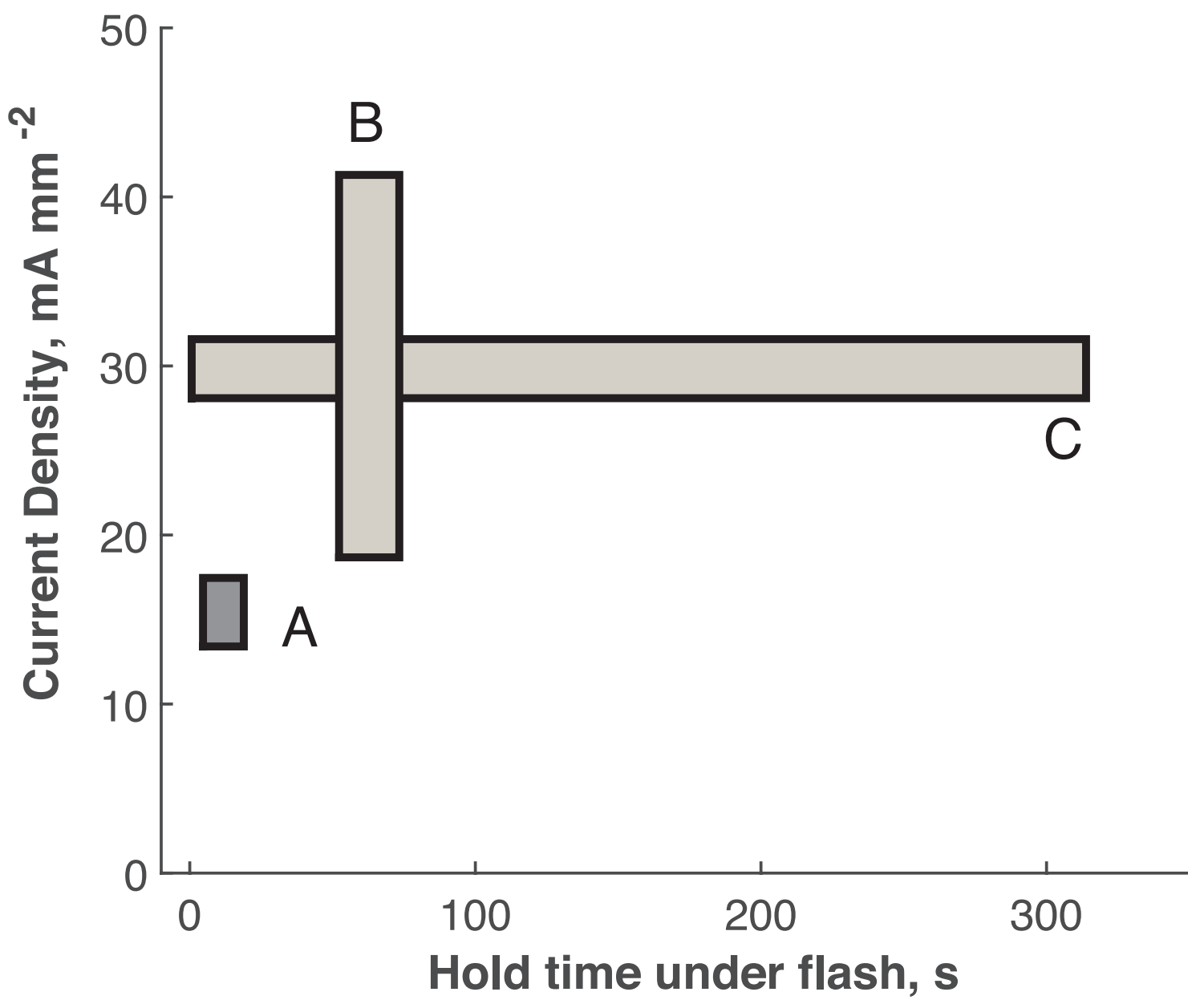

Figure 2. Experimental domains: $A$ is low current and low hold time for which no phase transformation was observed. $B$ is varying current density at a constant hold time. $C$ is variable hold time at current density of $30 \mathrm{~mA} \mathrm{~mm}^{-2}$. 


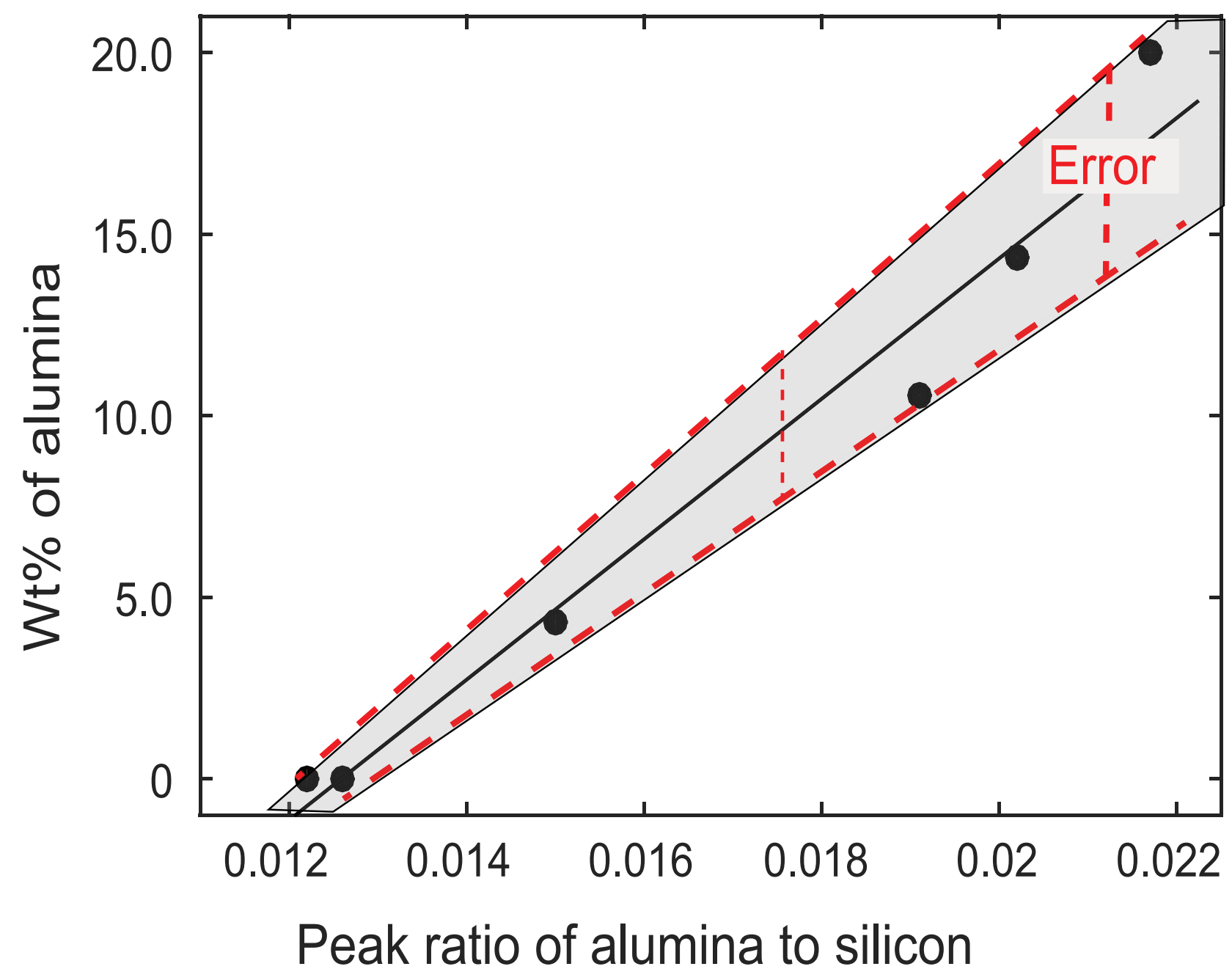

Figure 3. Calibration of the relative-intensity-ratio method for determining the weight fraction of alumina in the specimen, using silicon as the standard. These data were obtained by mixing powders of alumina and silicon with different weight fractions of alumina. 


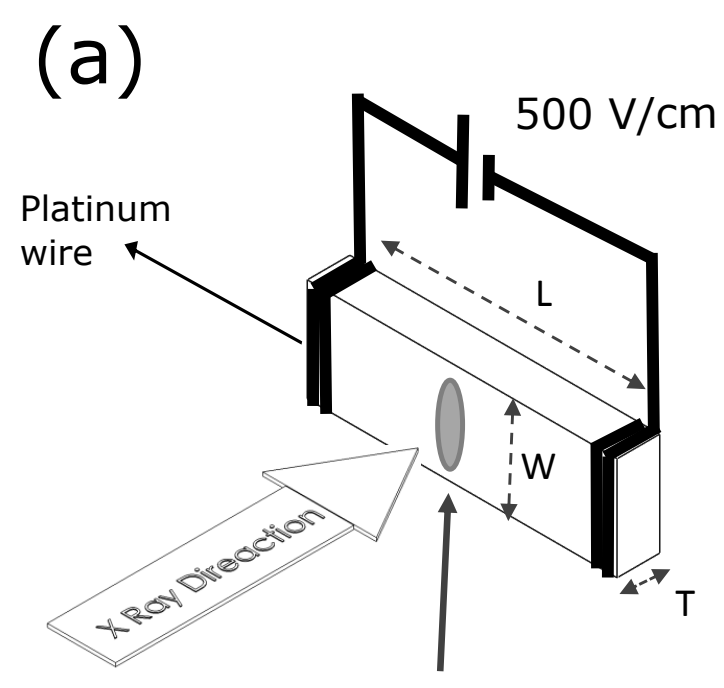

(b)

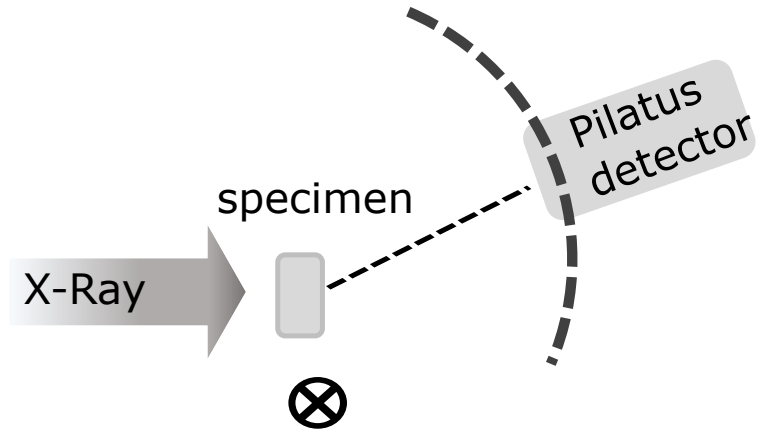

Electric field

Platinum paint

Figure 4. (a)The configuration of the set-up for in-situ experiments of $X$-ray diffraction at the APS. (b) The experiments were carried out in the transmission mode. 


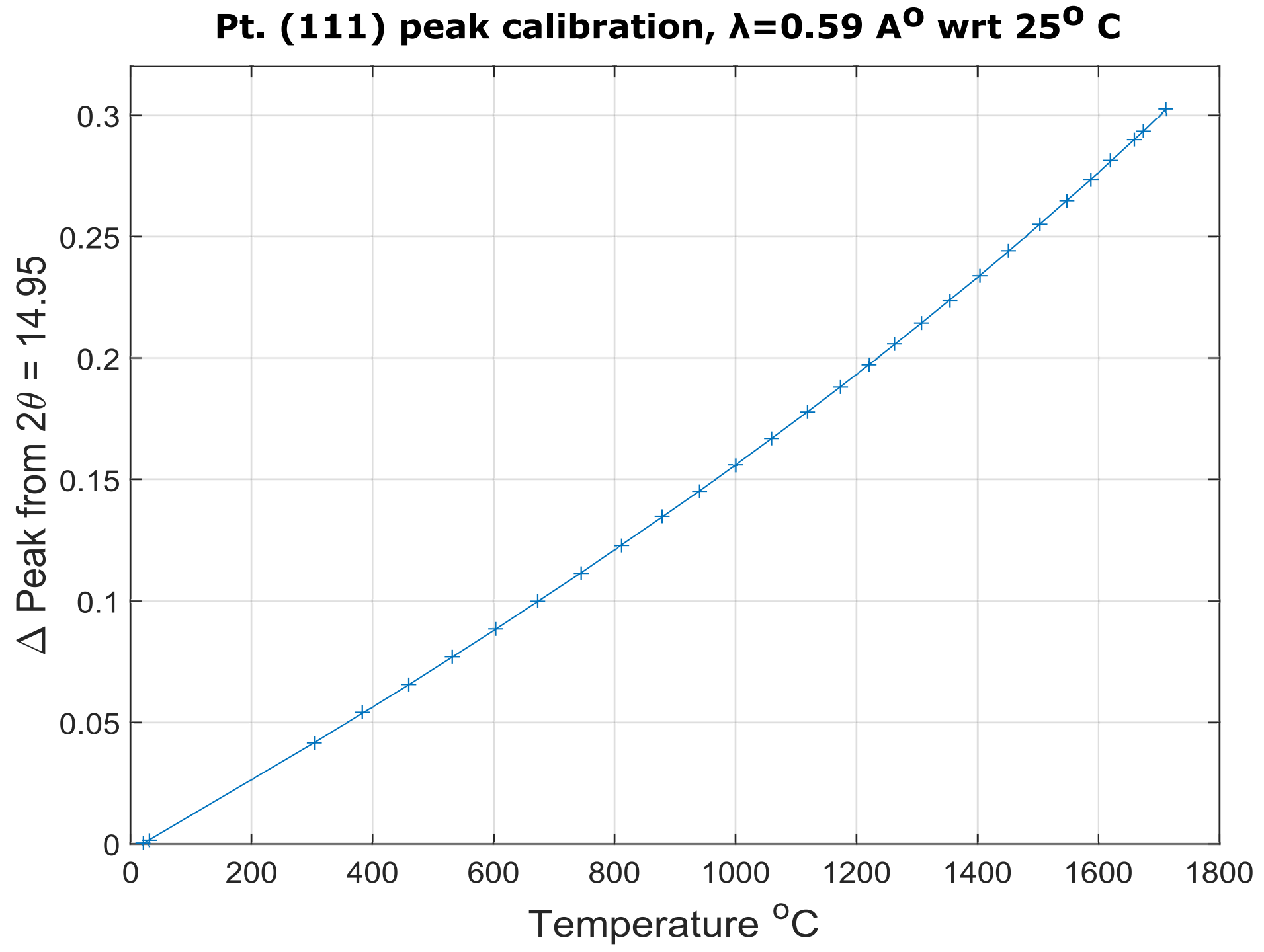

Figure 5. Platinum (111) peak shift has been calibrated with the temperature of specimen in conventional heating. 


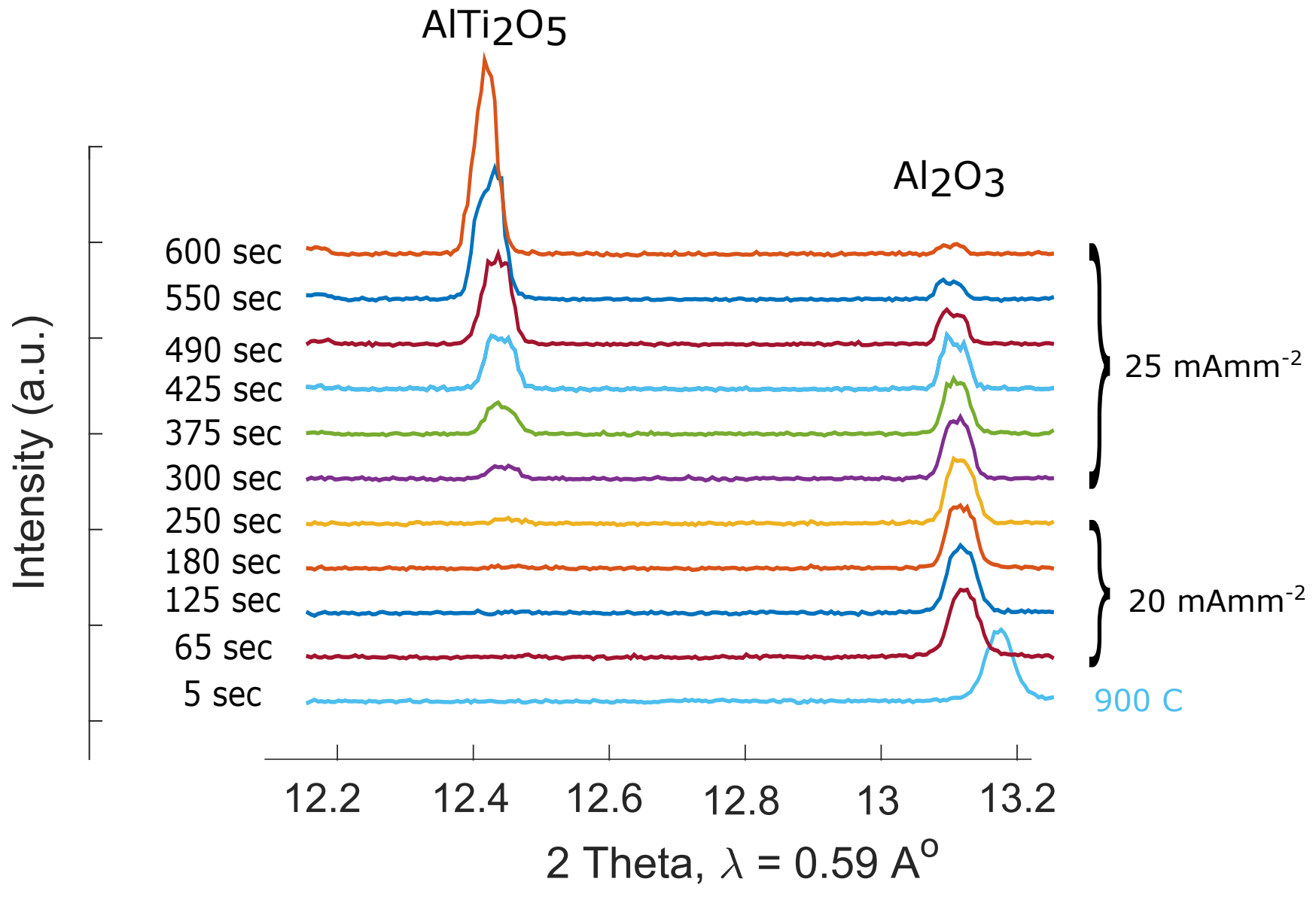

Figure 6. in-situ experiments of phase transformation in real time at the APS. The transformation is shown by dissolution of alumina and correspondingly the emergence of $\mathrm{Al}_{2} \mathrm{TiO}_{5}$ 

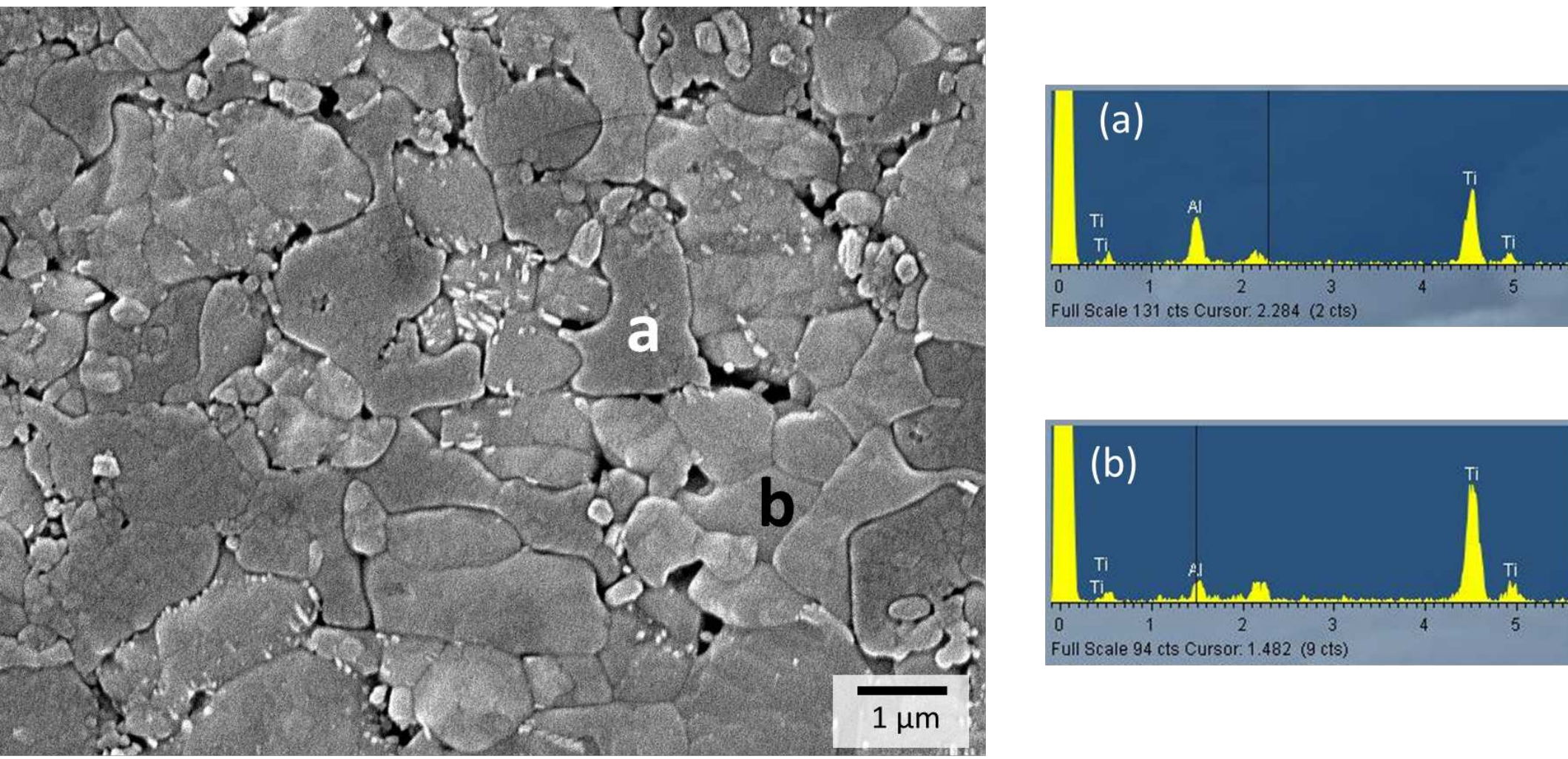

Figure 7. Micrograph of titania - aluminum titanate composite after the completion of reaction. EDS at two location shows two phase: titania rich and aluminum titanate. No pure alumina was found. 
(a)

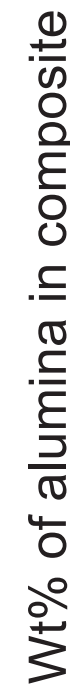

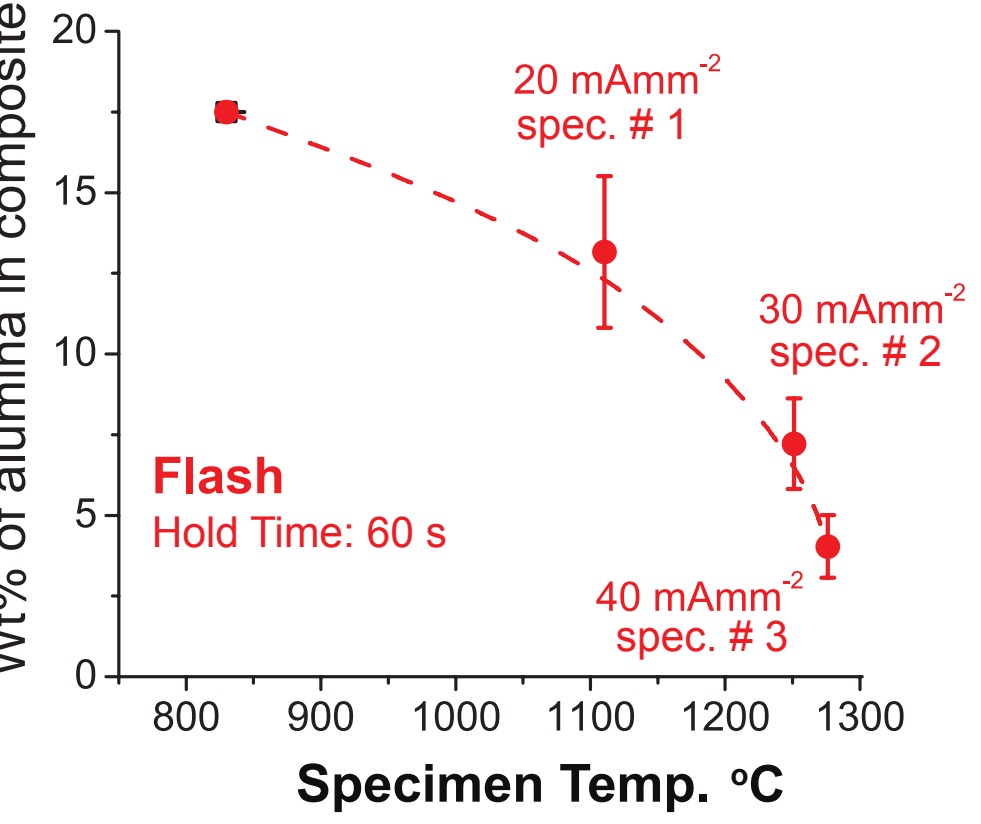

(b)

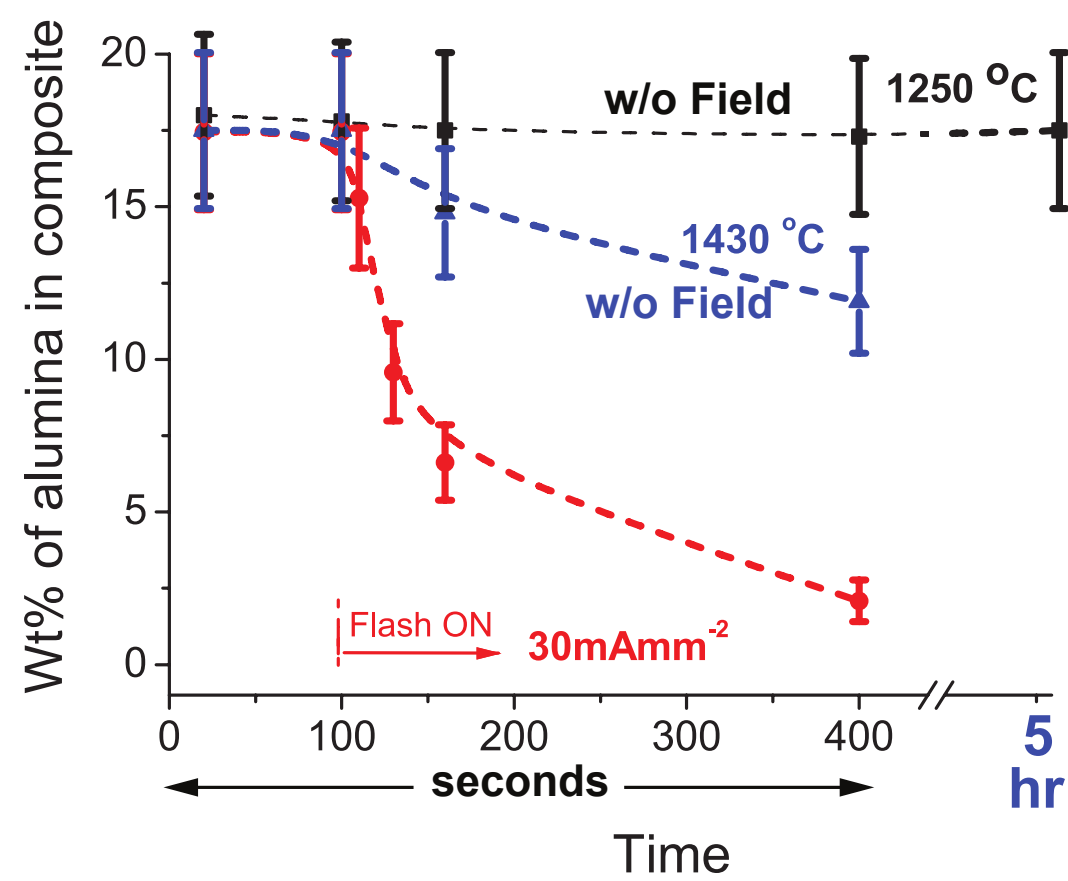

Figure 8. (a) The phase transformation reaction - shown by depletion of alumina - in three sepcimens held for $60 \mathrm{~s}$ at three different current densities. The specimen temperature is calculated from BBR model. (b) Phase transformation measured as a fuction of hold time at a current density of $30 \mathrm{mAmm}-2$. Includes, phase transformation at $1250{ }^{\circ} \mathrm{C}$, and at 1430 ${ }^{\circ} \mathrm{C}$ without electric field. The $1250^{\circ} \mathrm{C}$ corresponds to the specimen temperature at the current density of $30 \mathrm{mAmm}^{-2}$. 


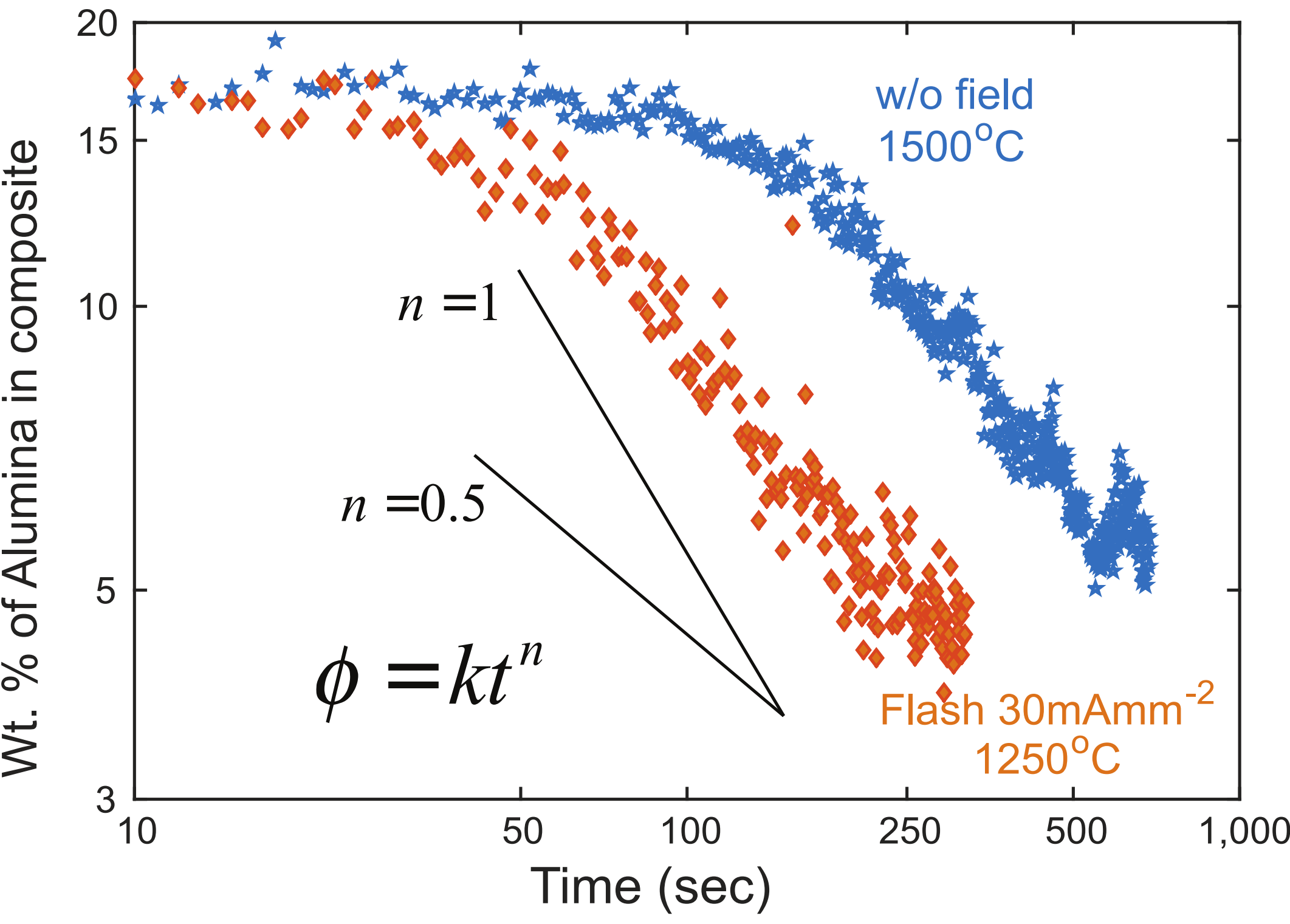

Figure 9. Log-log plot of alumina with hold time from live experiment at APS. 


\section{List of Figures}

1. Figure 1. A typical flash sintering experiment for composite $\mathrm{TiO}_{2}-\mathrm{Al}_{2} \mathrm{O}_{3}$ at a current density of $15 \mathrm{mAmm}^{-2}$. Stage I is the incubation stage, under voltage control, before the onset of the flash, the transient is Stage II during which time the sample sinters, and the following steady state of flash under current control is Stage III.

2. Figure 2. Experimental domains: A is low current and low hold time for which no phase transformation was observed. B is varying current density at a constant hold time. $\mathrm{C}$ is variable hold time at current density of $30 \mathrm{~mA} \mathrm{~mm}^{-2}$.

3. Figure 3. Calibration of the relative-intensity-ratio method for determining the weight fraction of alumina in the specimen, using silicon as the standard. These data were obtained by mixing powders of alumina and silicon with different weight fractions of alumina.

4. Figure 4. (a) The configuration of the set-up for in-situ experiments of X-ray diffraction at the APS. (b) The experiments were carried out in the transmission mode.

5. Figure 5. Platinum (111) peak shift has been calibrated with the temperature of specimen in conventional heating.

6. Figure 6. In-situ experiments of phase transformation in real time at the APS. The transformation is shown by dissolution of alumina and correspondingly the emergence of $\mathrm{Al}_{2} \mathrm{TiO}_{5}$.

7. Figure 7. Micrograph of titania- aluminum titanate composite after the completion of reaction. EDS at the two location shows two phases: titania rich and aluminum titanae. No pure alumina was found.

8. Figure 8. (a) The phase transformation reaction - shown by depletion of alumina - in three specimens held for $60 \mathrm{~s}$ at three different current densities. The specimen temperature is calculated from the BBR model. (b) Phase transformation measured at a function of the hold time at a current density of $30 \mathrm{~mA} \mathrm{~mm}^{-2}$. (b) Phase transformation at 
$1250{ }^{\circ} \mathrm{C}$, and at $1430^{\circ} \mathrm{C}$ without the electric field. The $1250{ }^{\circ} \mathrm{C}$ corresponds to the specimen temperature at the current density of $30 \mathrm{~mA} \mathrm{~mm}^{-2}$.

9. Figure 9. Log-log plot of alumina with hold time from live experiments at APS.

\section{List of tables}

Table 1. Black body radiation temperature calculations and comparison with in-situ measurements. 